\title{
Method for Performance Measurement of SoS Flow Response Time and Information Interaction
}

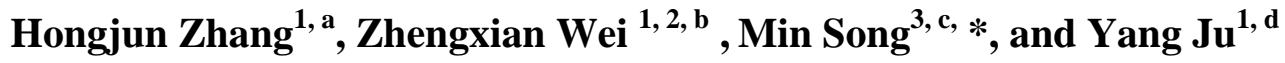 \\ ${ }^{1}$ Systems Engineering Research Institute, Beijing, China \\ ${ }^{2}$ Joint Laboratory for Smart Ocean Technology, Pilot National Laboratory for Marine Science and \\ Technology, Qingdao, China \\ ${ }^{3}$ Information Technology Centre, Beijing Foreign Studies University, Beijing, China \\ ${ }^{a}$ xiaobao2857@sina.com, ${ }^{b}$ weizhengxian@sina.com, ${ }^{c}$ songmin@bfsu.edu.cn, ${ }^{d}$ jyang-pioneer@163.com
}

Keywords: System-of-systems, process, response time, information interaction, performance measurement

\begin{abstract}
System-of-systems (SoS) is an organic whole that consists of different subsystems with the SoS flow dynamically established in the course of integration. The performance of SoS flow is an essential factor that affects the completion of SoS tasks. First, this paper proposes a response time computation model of SoS flow based on timed Petri Net, as well as establishing the methods for calculating the minimum and maximum response times of the flow branch paths. Through the transition probability of flow branches, a method for computing the average response time of SoS flow is established. Second, a measurement method for the performance of SoS flow information interaction is proposed based on the characteristics and requirements of information interaction among subsystems, and in terms of the completeness, accuracy and timeliness of information interaction. These efforts will enable the accurate computation of SoS flow performance in terms of response time and information interaction performance, thus providing the analysis and evaluation of the overall performance and effectiveness of SoS.
\end{abstract}

\section{Introduction}

The concept of 'system-of-systems' (SoS) has existed for a long time [1] and many scholars and organizations in different fields have proposed definitions of SoS from the backgrounds and perspectives of their respective fields. However, there is no generally accepted definition at present. SoS is the connection of systems. Mutual coordination and cooperation among systems are allowed in SoS connected by systems, such as C4I (Command, Control, Computers, Communications and Information) and ISR (Intelligence, Surveillance and Reconnaissance) systems in information battlefields [2]. SoS is the synthesis of systems. The purpose of system synthesis is the evolution, development, coordination and optimization of systems, reaching the aim of ultimately improving overall efficiency [3]. SoS is the integration of collaborative systems. These component systems have 
two additional characteristics, namely autonomy of operation and autonomy of management [4] [5]. United States Department of Defense: 'The capacity provided by the combination of interdependent systems is far greater than the sum capacity of those systems' [6]. The above definitions of SoS express its characteristics of large scale, complex structure and collaboration and integration by subsystems.

SoS integration requires the selection of appropriate subsystems to form an organic whole and the dynamic establishment of SoS flow according to specific task requirements [7]. The performance of dynamically established SoS flow becomes a key factor in the impact on the completion of SoS tasks. First, this paper proposes a timed Petri Net-based response time computation model of SoS flow. The data concept is introduced into the timed Petri Net (TPN) [8] [9]. As a response to the requirements of separation between data flow and control flow, a SoS flow model is established, and the methods for calculating the minimum and maximum response times of the flow branch paths are built. The SoS flow model is then converted into an isomorphic Markov chain [10] [11] and the average response time of the flow branches allows the computation of SoS flow through transition probability. Second, the implementation of SoS flow requires information interactions among different subsystems. A measurement method for the performance of SoS flow information interaction is proposed on the basis of the characteristics and requirements of information interaction among subsystems, and in terms of the completeness, accuracy and timeliness of information interaction. These efforts will enable the accurate computation of SoS flow performance in terms of response time and information interaction performance, thus providing the analysis and evaluation of the overall performance and effectiveness of SoS.

\section{Petri Net-based Response Time Computation Model of SoS Flow}

'SoS flow' means the temporal and logical relations executed in activities during the implementation of different tasks, and the resulting call process, data process and control process (including software and hardware subsystems and personnel). The purpose of SoS flow modeling is to allow the completion of relevant tasks with maximum efficiency. The process nature of SoS will determine the efficiency of task implementation, which requires the measurement of SoS flow performance. One major indicator that measures the performance of SoS flow is the process response time. A shorter response time indicates that SoS tasks are implemented more smoothly.

\subsection{TPN-based flow branch response time}

The response time of SoS flow should be measured using the maximum response time, minimum response time and average response time. Different processes may be executed for different states and different time intervals in the course of SoS task implementation. The maximum response time is the maximum time that SoS flow may experience from implementation to completion. The average response time means the average time in which SoS performs all processes.

For different tasks, SoS flow not only defines which activities should be executed but also determines a series of activity execution order, activity duration or activity start/end time as stringent conditions. Petri Net has a strong ability to describe system static models and analyze dynamic behavior. There is a 'start point' and an 'end point' in Petri Net. The place represents the condition, transition represents activity and the activity execution relation may be expressed in the four basic structures of sequence, selection, parallel and circulation. Such relations are linked by adding a place between two activities. TPN [9] links a time interval of implementation for each transition based on the classic Petri Net, which describes the asynchronism and concurrency flow. This is the best method for analyzing the activity flow and time constraints among SoS subsystems. The SoS flow 
model (SFM model) is established on the basis of TPN. The SFM model is centered on the characteristics of SoS subsystems that are loosely coupled in form and closely related in activity execution. It is capable of analyzing and calculating the maximum and minimum time consumption of the flow.

Information plays a critical role in the course of SoS task implementation, such as in the indication information of external targets. The flow will not continue until such information requirements are satisfied. As such, the data concept should be introduced in the course of modeling as a solution for the lack of data in Petri Net. It also supports the separation between data flow and control flow. The SoS flow model is defined with the generalization segments as shown in Figure 1:

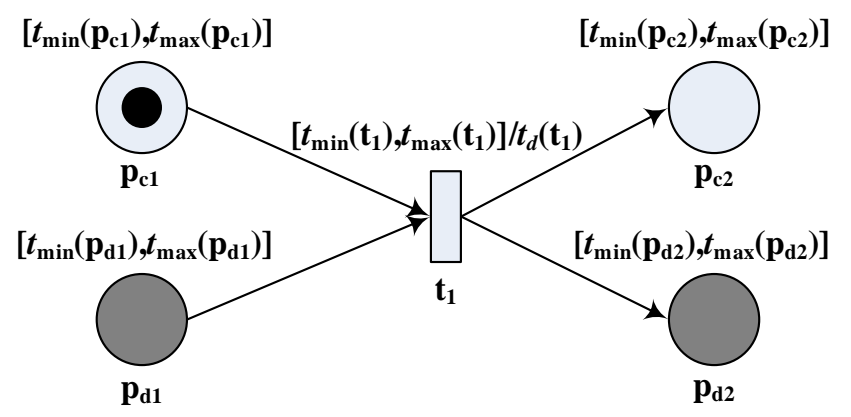

Fig. 1 Generalization Segment of SFM Model

The SoS flow model is an eight-element set of SFM=: $\left(P_{c}, P_{d}, T, F_{c}, F_{d}, C, D\right.$ and $\left.M\right)$, where: $\mathrm{P}_{\mathrm{c}}=\left\{\mathrm{p}_{\mathrm{c} 1}, \mathrm{p}_{\mathrm{c} 2}, \ldots, \mathrm{p}_{\mathrm{cm}}\right\}$ is the nonempty finite control place set;

$\mathrm{P}_{\mathrm{d}}=\left\{\mathrm{p}_{\mathrm{d} 1}, \mathrm{p}_{\mathrm{d} 2}, \ldots, \mathrm{p}_{\mathrm{dm}}\right\}$ is the nonempty finite data place set;

$\mathrm{F}_{\mathrm{C}} \subseteq \mathrm{P}_{\mathrm{C}} \times \mathrm{T} \cup \mathrm{T} \times \mathrm{P}_{\mathrm{c}}$ is the finite control arc set;

$\mathrm{F}_{\mathrm{d}} \subseteq \mathrm{P}_{\mathrm{d}} \times \mathrm{T} \cup \mathrm{T} \times \mathrm{P}_{\mathrm{d}}$ is the finite data arc set;

$\mathrm{C}$ is a set of real number pairs $\left[t_{\min }, t_{\max }\right]$ that relates place to transition;

$\mathrm{D}$ is a set of execution delay $t_{\mathrm{d}}(\mathrm{t})$ of transition;

$\mathrm{M}$ is the $\mathrm{m}$-dimension vector set (marking) used to describe the system state, where component $\mathrm{M}(\mathrm{p})$ represents the token number in place $\mathrm{p} . \mathrm{M}_{0}$ generally represents the initial marking.

The operation of SoS flow is always controlled by the control flow in SFM. However, data flow provides necessary data support for the operation of control flow. It is assumed that there is a control token in the SFM model. In SFM, however, $\left[t_{\min }\left(\mathrm{p}_{\mathrm{c} 1}\right), \mathrm{t}_{\max }\left(\mathrm{p}_{\mathrm{c} 1}\right)\right]$ and $\left[t_{\min }\left(\mathrm{p}_{\mathrm{c} 2}\right), t_{\max }\left(\mathrm{p}_{\mathrm{c} 2}\right)\right]$ are local time constraints of places $\mathrm{p}_{\mathrm{c} 1}$ and $\mathrm{p}_{\mathrm{c} 2}$ in terms of control flow. They represent the enabled interval in which the control place supports the occurrence of subsequent transitions. For example, if place $\mathrm{p}_{\mathrm{c} 1}$ obtains a token at $T_{0}, \mathrm{t}_{1}$ will be enabled in interval $\left[T_{0}+t_{\min }\left(\mathrm{p}_{\mathrm{c} 1}\right), T_{0}+t_{\max }\left(\mathrm{p}_{\mathrm{c} 1}\right)\right]$ in the control flow.

SoS subsystems are loosely connected. The data to be used may not be obtained at the right time. During the use of data, the times at which data arrives at and leaves the place are the local time constraints of data places $\mathrm{p}_{\mathrm{c} 1}$ and $\mathrm{p}_{\mathrm{c} 2}$, and represent the enabled interval in which the data place supports the occurrence of subsequent transitions (based on the fact that the control place support transition enabling).

Transition activity execution requires the execution time interval and execution time delay. [ $\left.\left.t_{\min }\left(\mathrm{t}_{1}\right), t_{\max }\left(\mathrm{t}_{1}\right)\right] / t_{d}\left(\mathrm{t}_{1}\right)\right]$ is the local time constraint at transition $\mathrm{t}_{1}$. The former represents the triggerable interval of transition $t_{1}$. The latter represents the execution delay interval of time $t_{1}$. Assuming that transition $t_{1}$ is enabled at $T_{1}$, it will be triggered in interval $\left[T_{1}+t_{\min }\left(\mathrm{t}_{1}\right), T_{1}+t_{\max }\left(\mathrm{t}_{1}\right)\right]$ only due to the local time constraint of transition.

The input/output place set of transition t may be expressed in $I_{\mathrm{p}}(\mathrm{t}) / O_{\mathrm{p}}(\mathrm{t}) ; I_{\mathrm{p}}(\mathrm{p}) / O_{\mathrm{p}}(\mathrm{p})$ denotes the input/output transition set of place $\mathrm{p} ; \delta=\left(\mathrm{M}_{0} \mathrm{t}_{1} \mathrm{M}_{1} \ldots \mathrm{t}_{i} \mathrm{M}_{i} \ldots \mathrm{t}_{n} \mathrm{M}_{n}\right)$ denotes that SoS flow goes from 
state $\mathrm{M}_{0}$ to state $\mathrm{M}_{n} ; \delta_{k}\left(\mathrm{M}_{n}\right)$ denotes the sequence of all places and transitions (except transition 1) on path $k$ from $\mathrm{M}_{0}$ to $\mathrm{M}_{n}$, where $n$ is the number of the last transition in the sequence; $t_{E E}(\mathrm{t}) / t_{L E}(\mathrm{t})$ denotes the first/last enable time of transition $\mathrm{t} ; t_{E F}(\mathrm{t}) / t_{L F}(\mathrm{t})$ denotes the earliest/latest triggerable time of transition $\mathrm{t}$; and $t_{E B}(\mathrm{t}) / t_{F E}(\mathrm{t})$ denotes the start/end time at which transition $\mathrm{t}$ is triggered. This can be expressed as follows:

$$
\begin{gathered}
{\left[t_{E B}(\mathrm{t}), t_{F E}(\mathrm{t})\right] \subseteq\left[t_{E F}(\mathrm{t}), t_{L F}(\mathrm{t})\right] \subseteq\left[t_{E E}(\mathrm{t}), t_{L E}(\mathrm{t})\right]} \\
t_{F E}(\mathrm{t})-t_{E B}(\mathrm{t})=t_{d}(\mathrm{t})
\end{gathered}
$$

With SFM modeling, the response time of SoS flow may be calculated. The computation first goes to the response time of each path and the average response time of the entire flow as a whole. The SFM model of a SoS is presented in Fig. 2.

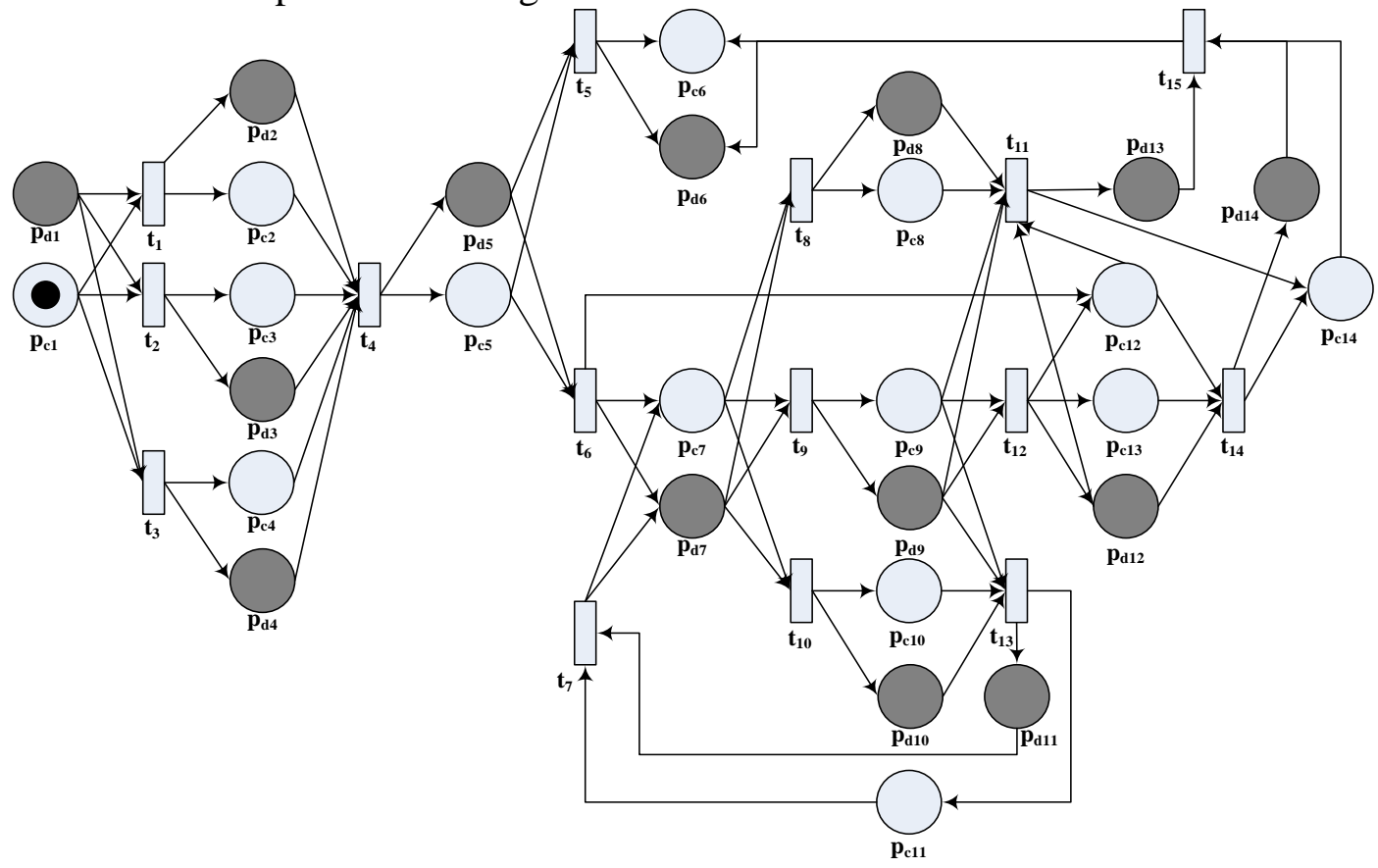

Fig. 2 SoS Flow Model

Each state $\mathrm{M}_{j}$ represents the state of the SoS flow. Each transition $\mathrm{t}_{j}$ represents a flow activity. The maximum and minimum response times of the path may be calculated due to the presence of time constraints on place and transition in the SoS SFM model. It is given as follows:

$$
\begin{aligned}
& \operatorname{Min}\left(T_{f}\right)=\sum_{i=0}^{n-1}\left\{\max \left(t_{\text {min }}\left(\mathrm{p}_{\mathrm{c} i}\right), t_{\text {min }}\left(\mathrm{p}_{\mathrm{d} i}\right)+\left[t_{\text {min }}\left(\mathrm{t}_{i}\right)+t_{d}\left(\mathrm{t}_{i}\right)\right]\right\}+T_{0}\right. \\
& \operatorname{Max}\left(T_{f}\right)=\sum_{i=0}^{n-1}\left\{\min \left(t_{\text {max }}\left(\mathrm{p}_{\mathrm{c} i}\right), t_{\text {max }}\left(\mathrm{p}_{\mathrm{d} i}\right)+\left[t_{\text {max }}\left(\mathrm{t}_{i}\right)+t_{d}\left(\mathrm{t}_{i}\right)\right]\right\}+T_{0}\right.
\end{aligned}
$$

Different tasks will be implemented in different intervals as the state changes during the implementation of SoS tasks. This results in the changeover of paths in the operational flow. The average response time of SoS flow may be calculated with consideration of the sum of transition probability of branch paths in the flow. 


\subsection{Markov chain-based flow average response time}

Flow changeover may occur whenever the state changes in the course of flow execution. The SFM model may be converted into an isomorphic Markov chain and the average response time of the flow branches allows the computation of SoS flow through transition probability. An SFM model is presented in Figure 3. The isomorphic Markov chain is presented in Fig. 4.

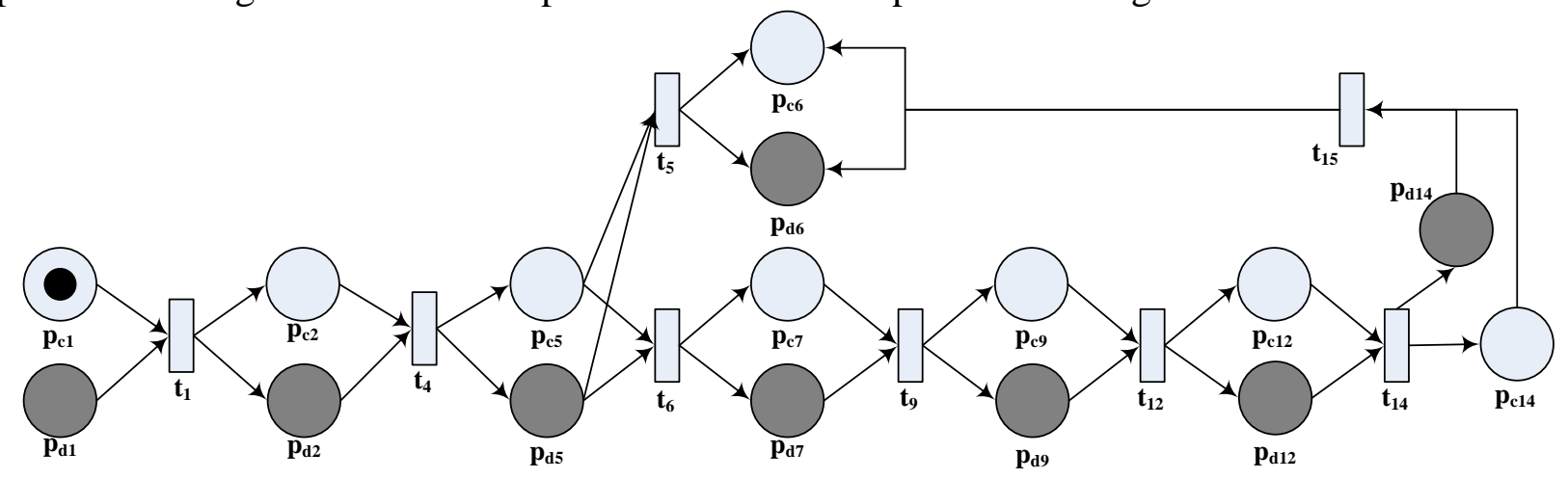

Fig. 3 Example of an SFM Model

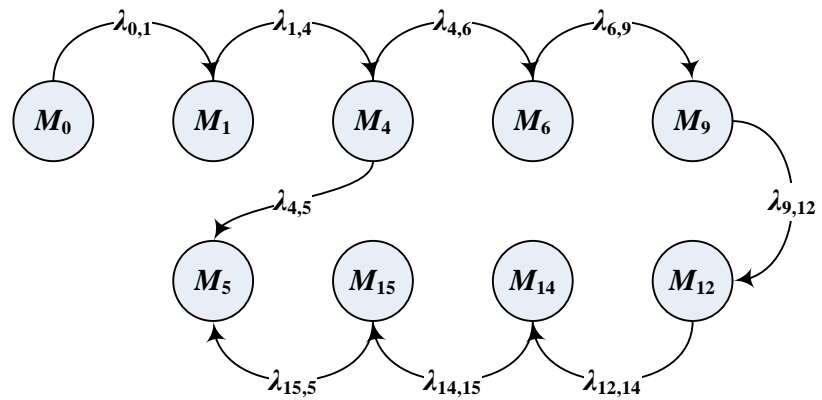

Fig. 4 Isomorphic Markov Chain of the SFM Model

The state transition matrix $Q$ is established according to the isomorphic Markov chain. When there is an arc for connection from state $M_{i}$ to state $M_{j}$, it means that state $M_{i}$ can be transitioned to state $M_{j}$. Element $\lambda_{i, j}$ in $Q$ represents the probability of transition from $M_{i}$ to $M_{j}$. When there is no arc for connection from state $M_{i}$ to state $M_{j}$, element $\lambda_{i, j}$ in $Q$ will be 0 . The element on the diagonal of state transition matrix $Q$ is 1 , which means that the internal transition probability of the state is always 1. The state transition matrix of the isomorphic Markov chain for the SFM model is presented in the figure below:

$$
Q=\left[\begin{array}{ccccc}
1 & \lambda_{0,1} & \lambda_{0,2} & \cdots & \lambda_{0, n-1} \\
\lambda_{1,0} & 1 & \lambda_{1,2} & \cdots & \lambda_{1, n-1} \\
\vdots & \vdots & \ddots & \vdots & \vdots \\
\lambda_{n-2,0} & \lambda_{n-2,1} & \cdots & 1 & \lambda_{n-2, n-1} \\
\lambda_{n-1,0} & \lambda_{n-1,1} & \cdots & \cdots & 1
\end{array}\right]
$$

The average response time of SoS flow is the average time it takes to start from $M_{0}$, pass through all possible branches and arrive at the final state $M_{e}$. In this process, the average minimum and maximum response times it takes to transfer from the former to current state $M_{i}$ are: 


$$
\begin{aligned}
& \operatorname{AveMin}\left(\mathrm{M}_{i}\right)=\left(\max \left(t_{\min }\left(\mathrm{p}_{\mathrm{c} i}\right), t_{\min }\left(\mathrm{p}_{\mathrm{d} i}\right)+\left[t_{\min }\left(\mathrm{t}_{i}\right)+t_{d}\left(\mathrm{t}_{i}\right)\right]\right) \lambda_{i-1, i}\right. \\
& \operatorname{AveMax}\left(\mathrm{M}_{i}\right)=\left(\min \left(t_{\max }\left(\mathrm{p}_{\mathrm{c} i}\right), t_{\max }\left(\mathrm{p}_{\mathrm{d} i}\right)+\left[t_{\max }\left(\mathrm{t}_{i}\right)+t_{d}\left(\mathrm{t}_{i}\right)\right]\right) \lambda_{i-1, i}\right.
\end{aligned}
$$

The average minimum and maximum response times of SoS flow are:

$$
\begin{aligned}
& \operatorname{AveMin}\left(T_{f}\right)=\frac{\sum_{i=1}^{n} \operatorname{AveMin}\left(\mathrm{M}_{i}\right)}{n} \\
& \operatorname{AveMax}\left(T_{f}\right)=\frac{\sum_{i=1}^{n} \operatorname{AveMax}\left(\mathrm{M}_{i}\right)}{n}
\end{aligned}
$$

\section{Analysis and Computation of the Performance of SoS Flow Information Interaction}

SoS comprises different subsystems. Information interaction is required in the course of SoS flow execution. The performance of information interaction greatly affects that of SoS flow. The completeness, accuracy and timeliness of the information interaction of SoS systems are main factors affecting the performance of SoS flow information interaction. The analysis and computation of information interaction performance is based on these factors.

It is assumed that in SoS flow, information interaction exists in subsystems $M_{1}$ and $M_{2}$. During the interval of both information interactions, $M_{1}$ serves as the information sender, with the information $Q$ updated as follows:

$$
Q=\left[Q\left(t_{1}\right) Q\left(t_{2}\right) \cdots Q\left(t_{k_{2}}\right)\right]=\left[\begin{array}{cccc}
q_{1}\left(t_{1}\right) & q_{1}\left(t_{2}\right) & \cdots & q_{1}\left(t_{k_{2}}\right) \\
q_{2}\left(t_{2}\right) & q_{2}\left(t_{2}\right) & \cdots & q_{2}\left(t_{k_{2}}\right) \\
\vdots & \vdots & \ddots & \vdots \\
q_{k_{1}}\left(t_{1}\right) & q_{k_{1}}\left(t_{2}\right) & \cdots & q_{k_{1}}\left(t_{k_{2}}\right)
\end{array}\right]
$$

$M_{2}$ is the information receiver, with the information $Q^{\prime}$ received from $M_{1}$ as follows:

$$
Q^{\prime}=\left[\begin{array}{llll}
Q^{\prime}\left(t_{1}^{\prime}\right) & Q^{\prime}\left(t_{2}^{\prime}\right) \cdots & Q^{\prime}\left(t_{k_{2}}^{\prime}\right)
\end{array}\right]=\left[\begin{array}{cccc}
q_{1}^{\prime}\left(t_{1}^{\prime}\right) & q_{1}^{\prime}\left(t_{2}^{\prime}\right) & \cdots & q_{1}^{\prime}\left(t_{k_{2}}^{\prime}\right) \\
q_{2}^{\prime}\left(t_{2}^{\prime}\right) & q_{2}^{\prime}\left(t_{2}^{\prime}\right) & \cdots & q_{2}^{\prime}\left(t_{k_{2}}^{\prime}\right) \\
\vdots & \vdots & \ddots & \vdots \\
q_{k_{1}}^{\prime}\left(t_{1}^{\prime}\right) & q_{k_{1}}^{\prime}\left(t_{2}^{\prime}\right) & \cdots & q_{k_{1}}^{\prime}\left(t_{k_{2}}^{\prime}\right)
\end{array}\right]
$$

Where $t_{i}^{\prime} \geq t_{i}, i=1,2, \cdots, t_{k_{2}}$ and there may be blank rows in $Q^{\prime}$ expressed in \#.

\subsection{Completeness of information interaction}

The completeness of information interaction is the consistency between the information content received by subsystem $M_{2}$ and that sent by subsystem $M_{1}$ in the SoS flow. The measurement equation is: 


$$
v_{f}=\frac{1}{k_{2}} \sum_{i=1}^{k_{2}} \Delta_{1}\left(Q^{\prime}\left(t_{i}^{\prime}\right)\right)
$$

Where $\Delta_{1}\left(Q^{\prime}\left(t_{i}^{\prime}\right)\right)=\left\{\begin{array}{l}1, Q^{\prime}\left(t_{i}^{\prime}\right) \neq \# \\ 0, Q^{\prime}\left(t_{i}^{\prime}\right)=\#\end{array}\right.$, it is obvious that there is $0 \leq v_{f} \leq 1$ and a greater $v_{f}$ value indicates less loss of information.

\subsection{Accuracy of information interaction}

The accuracy of information interaction is the consistency between the information content received by subsystem $M_{2}$ and that sent by subsystem $M_{1}$ in the SoS flow. The measurement equation is:

$$
v_{e}=\frac{1}{k_{1} \sum_{i=1}^{k_{2}} \Delta_{1}\left(Q^{\prime}\left(t_{i}^{\prime}\right)\right)} \sum_{\substack{j=1,2, \ldots, k_{2} \\ Q^{\prime}\left(t_{j}^{\prime}\right) \neq \#}}\left(\sum_{i=1}^{k_{1}} \Delta_{2}\left(q_{i}\left(t_{j}\right), q_{i}^{\prime}\left(t_{j}^{\prime}\right)\right)\right)
$$

Where $\Delta_{2}\left(q_{i}\left(t_{j}\right), q_{i}^{\prime}\left(t_{j}^{\prime}\right)\right)=\left\{\begin{array}{l}1, q_{i}\left(t_{j}\right)=q_{i}^{\prime}\left(t_{j}^{\prime}\right) \\ 0, q_{i}\left(t_{j}\right) \neq q_{i}^{\prime}\left(t_{j}^{\prime}\right)\end{array}\right.$, it is obvious that there is $0 \leq v_{e} \leq 1$ and a greater $v_{e}$ value indicates less external interference on information content.

\subsection{Timeliness of information interaction}

The timeliness of information interaction is the conformity of the information interaction time of subsystems in the SoS flow. The measurement equation is:

$$
v_{t}=\frac{\operatorname{card}\left(\left\{i=1,2, \cdots, k_{2} \| \tau_{i}-\left(t_{i}^{\prime}-t_{i}\right) \mid \leq \delta\left(\tau_{i}\right), Q^{\prime}\left(t_{i}^{\prime}\right) \neq \#\right\}\right)}{\sum_{i=1}^{k_{2}} \Delta_{1}\left(Q^{\prime}\left(t_{i}^{\prime}\right)\right)}
$$

Where $\tau_{i}$ represents the interaction time delay of information interaction at $t_{i}, \delta\left(\tau_{i}\right)$ represents the maximum allowable deviation from the interaction time delay of the subsystem at $t_{i}$ and $\operatorname{card}(\bullet)$ represents the number of set elements, namely the amount of information for interaction. It is obvious that there is $0 \leq v_{t} \leq 1$, and a greater $v_{t}$ value indicates that the transmission time delay among subsystems meets the requirements more fully.

The completeness, accuracy and timeliness may be defined as the average level of completeness, accuracy and timeliness of information interaction between subsystems when the information interaction among several subsystems in the SoS flow is considered.

\section{Conclusion}

A system-of-systems (SoS) is an organic whole that consists of different subsystems with the SoS flow dynamically established in the course of integration. The performance of dynamically established SoS flow becomes a key factor having an impact on the completion of SoS tasks. Based on TPN, a SoS flow model is established, the methods for calculating the minimum and maximum response times of the flow branch paths are built, and the response time computation model of SoS 
flow is proposed. The SoS flow model is then converted into an isomorphic Markov chain and the average response time of the flow branches allows the computation of SoS flow through transition probability. A measurement method for the performance of SoS flow information interaction is proposed on the basis of the characteristics and requirements of information interaction among subsystems, and in terms of the completeness, accuracy and timeliness of information interaction. These efforts will enable the accurate computation of SoS flow performance in terms of response time and information interaction performance, thus providing the analysis and evaluation of the overall performance and effectiveness of SoS.

\section{Acknowledgments}

This work is sponsored by the National Natural Science Foundation of China under Grant Nos. 61502037 and 61772152, Basic Research Project Nos. JCKY2016206B001, JCKY2014206C002 and JCKY2016604C010, and the Foundation of Joint Laboratory for Smart Ocean Technology. Corresponding author: Min Song.

\section{References}

[1] Lane J A, Valerdi R. Synthesizing SoS Concepts for Use in Cost Modeling [J]. Systems Engineering, 2007, 10(4): 297-308.

[2] Manthorpe Jr., W.H. 'The Emerging Joint System-of-Systems: A Systems Engineering Challenge and Opportunity for APL,' John Hopkins APL Technical Digest, Vol. 17, No. 3 (1996), pp. 305-310.

[3] Pei, R.S. 'Systems-of-Systems Integration (SoSI)—A Smart Way of Acquiring Army C4I2WS Systems,' Proceedings of the Summer Computer Simulation Conference, (2000), pp. 574-579.

[4] Sage, A.P. and C.D. Cuppan. 'On the Systems Engineering and Management of Systems-of-Systems and Federations of Systems,’ Information, Knowledge, Systems Management, Vol. 2, No. 4, 2001, pp. 325-345.

[5] Maier M W. Architecting Principles for Systems-of-Systems [J]. System Engineering, 1998, 1(4):267-284.

[6] GAO. Defense Acquisitions DoD Management Approach and Processes Not-Well Suited to Supports Development of Global Information Grid,’ January 2006.

[7] C. Keating et al. 'Systems of Systems Engineering'. Engineering Management Journal. Vol. 15, NO. 2, pp. 32-41. Jun. 2003.

[8] Pelz, Elisabeth. 'Full Axiomatisation of Timed Processes of Interval-Timed Petri Nets.' Fundamenta Informaticae 157.4(2018):427-442.

[9] Huang, Yi-Sheng, Y. Weng, and M. C. Zhou. 'Design of Regulatory Traffic Light Control Systems with Synchronized Timed Petri Nets.’ Asian Journal of Control 20.5(2018).

[10] Guan, Ji, Y. Feng, and M. Ying. 'Decomposition of quantum Markov chains and its applications.' Journal of Computer \& System Sciences (2018).

[11] Poznanović, Svetlana, and K. Stasikelis. 'Properties of the promotion Markov chain on linear extensions.' Journal of Algebraic Combinatorics 4(2017):1-24. 